\title{
The projection factor, period-radius relation, and surface-brightness colour relation in classical cepheids
}

\author{
M. A. T. Groenewegen
}

Instituut voor Sterrenkunde, Celestijnenlaan 200 D, 3001 Leuven, Belgium

e-mail: groen@ster.kuleuven.be

Received 5 July 2007 / Accepted 14 August 2007

ABSTRACT

\begin{abstract}
Context. The projection factor ( $p$-factor) is of crucial importance in linking radial velocity variations to radius variations in radially variable stars such as RR Lyrae of Cepheids. In turn, this is a crucial ingredient in the Baade-Wesselink method in obtaining distances to these stars using a surface-brightness (SB) colour relation.

Aims. Our aim is to establish a relation between the $p$-factor and pulsation period based on six cepheids with interferometrically measured angular diameter variations and known distances. As a by-product, a period-radius (PR) relation is derived which in turn is used to derive the $p$-factor and distance for two stars. In addition, the data allows calibration of the SB colour relation.

Methods. Literature values of the $V$-band, $K$-band and radial velocity curves are collected and fitted with Fourier series. For stars with known distances and measured angular diameters as a function of the pulsation phase, values for the radius and the $p$-factor can be obtained. A PR relation is derived based on five stars. For two cepheids, this PR relation is used to determine the radius and then solve for the $p$-factor and the distance. From the Fourier series, the $V$ and $K$ values at the times of the angular diameter measurements are derived, and the SB colour relation can be established. Allowance is made for the recent discovery of circumstellar material around some cepheids which influences both the derived angular diameters and the colours.

Results. The PR relation derived is: $\log R=0.686 \log P+1.134$, slightly shallower than recently discussed PR-relations in the literature but in agreement with theory. Based on a total of eight stars with periods in the range 5-35 days there is no evidence that the $p$-factor depends on period, and the best-fitting constant value is $p=1.27 \pm 0.05$. The $\mathrm{SB}$ relation derived is $\log \theta_{0}=$ $0.275(V-K)_{0}+0.524$, in excellent agreement with that derived by Kervella et al. (2004b, A\&A, 428, 587).
\end{abstract}

Key words. stars: distances - Cepheids - distance scale - stars: oscillations

\section{Introduction}

Obtaining accurate distances to stars is a non-trivial matter. Cepheids are considered an important standard candle as they are bright and are thus the link between the distance scale in the nearby universe and that further out via those galaxies that contain both Cepheids and SNIa.

Distances to local cepheids may be obtained via mainsequence fitting for those Cepheids in clusters or via determination of the parallax. Until recently only Polaris had an accurate parallax determination via Hipparcos. Benedict et al. (2007) recently published absolute trigonometric parallaxes for nine Galactic Cepheids using the Fine Guidance Sensor on board the Hubble Space Telescope, and revised Hipparcos parallaxes will soon become available (van Leeuwen et al. 2007).

In parallel, accurate interferometrically determined angular diameters over the pulsation phase have also become available over the last few years (e.g. Kervella et al. 2004a; Mérand et al. 2005), clearly improving on earlier work (e.g. Nordgren et al. 2002).

These new developments allowed Mérand et al. (2005) to derive the projection factor ( $p$-factor) in $\delta$ Cep using the parallax distance from Benedict et al. (2002). The newly available angular diameter measurements also allowed Kervella et al. (2004b) to calibrate the surface-brightness (SB) colour relation for Cepheids. Both the $p$-factor and SB colour relations are crucial ingredients in applying the Baade-Wesselink technique in obtaining distances to Cepheids.
In this paper I revisit the determination of the $p$-factor and the SB $(V-K)$ colour relation using the currently available sample of Cepheids with accurate published trigonometric parallaxes and angular diameter measurements, also considering the recent discovery of the presence of extended emission around a few Cepheids (e.g. Kervella et al. 2006), which might influence the derived angular diameters as well as the observed colours.

Section 2 describes the selection of the photometric, radial velocity and interferometric data. Section 3 outlines how the data is modelled, and Sects. 4, 5, 6, describe the results for the PR relation, the $p$-factor and the SB relation, respectively. Section 7 presents conclusions.

\section{The data}

For the objects studied in the present paper, Table 1 lists the sources of the $V, K$ and radial velocity $(R V)$ data considered in this study. The McMaster Cepheid Photometry and Radial Velocity Data Archive ${ }^{1}$ was a good start for searching for data, and also the database on Cepheids in binary systems ${ }^{2}$ (Szabados 2003) is useful.

In a first step, in an attempt to homogenise the datasets, the light curves are read in the program Period04 (Lenz \& Breger 2005), which allows for an easy visualisation of different datasets. Period04 is used to calculate a period and phase the

\footnotetext{
${ }^{1}$ http://crocus.physics.mcmaster.ca/Cepheid/

2 http://www. konkoly.hu/CEP/intro.html
} 
Table 1. Sources of $V$-, $K$-band and RV data.

\begin{tabular}{cccc}
\hline \hline Name & $V$ & $K$ & $R V$ \\
\hline delta Cep & $7(+0.01), 8,9(-0.06), 10(-0.04), 11,12(+0.03)$ & 10 & $1,2,3(+3.0), 4(-2.0), 5(-2.0), 6(-1.4)$ \\
l Car & $8,17,18,7,19$ & 20 & $13(+6.0), 8(+2.0), 14,15,16$ \\
zeta Gem & $7,22,11(-0.03), 9(+0.03), 18$ & 21 & $16(-0.9), 3,2(+1.2), 1,4,23$ \\
beta Dor & $7(+0.01), 8,19$ & 20,38 & $8,13,16,36,37$ \\
W Sgr & $7,11(-0.01), 28,31$ & $21,24,25$ & $1(+0.1), 2(-0.7), 3(-3.2), 13(+0.6)$, \\
& & & $26(-1.0), 27(-0.7), 28,29(+1.0), 30(+1.6)$ \\
X Sgr & $7,8(+0.01), 19,11,18$ & 21,24 & $13(+1.0), 3(-2.0), 2(-2.0), 8(+4.7), 32(+0.9)$, \\
& & & $33(-1.7), 34(+0.3)$ \\
eta Aql & $(+0.01), 9(+0.01), 10(-0.02), 11,12(-0.01), 18,19$ & $10,24(-0.01)$ & $2(+5.0), 3,4,5(+0.5), 6,13.35$ \\
Y Oph & $7,11(-0.02), 19(-0.03), 39$ & $20,24(-0.04)$ & $2,3,13,16,23,39,40,41,42$ \\
Y Sgr & $7,8(+0.02), 11,18,19(-0.02)$ & 21,24 & $2(-2.3), 3(-3.0), 8,16(-2.5), 13(-1.5)$ \\
FF Aql & $7,8,9,11,19,44(-0.02), 45(-0.03)$ & $21,24,38$ & $2(+12.0), 3(-0.7), 4(-0.7), 13(-0.6), 23,43(+0.5)$ \\
T Vul & $7,9(-0.01), 10,11,45$ & $10,21(+0.02), 24(-0.02)$ & $1,2,3(-2.2), 4(-2.2), 6(-1.8)$ \\
RT Aur & $9,10,11,45$ & 10,21 & $2,3,4,23$ \\
\hline
\end{tabular}

References: 1 = Bersier et al. (1994); 2 = Wilson et al. (1989); 3 = Barnes et al. (1987), points with uncertainty flag ":” were removed; 4 = Kiss (2000); 5 = Storm et al. (2004); 6 = Barnes et al. (2005); 7 = Berdnikov et al. (2000), a datafile named "cepheids-16-03-2006" was retrieved from the ftp address listed in that paper; $8=$ Bersier (2002), datapoints with weight 0 and 1 in the Geneva photometry were removed; $9=$ Kiss (1998); $10=$ Barnes et al. (1997); 11 = Moffett \& Barnes (1984); $12=$ Szabados (1980); 13 = Lloyd Evans (1980b); $14=$ Taylor et al. (1997); 15 = Caldwell et al. (2001); 16 = Nardetto et al. (2006); 17 = Madore (1975); $18=$ Shobbrook (1992); $19=$ Pel (1976); $20=$ Laney \& Stobie (1992); 21 = Wisniewski \& Johnson (1968); 22 = Szabados (1981); 23 = Gorynya et al. (1998, VizieR On-line Data Catalog: III/229); 24 = Welch et al. (1984); 25 = Kimeswenger et al. (2004); 26 = Petterson et al. (2004); 27 = Albrow \& Cottrell (1996); $28=$ Babel et al. (1989); $29=$ Jacobsen et al. (1984); 30 = Jacobsen (1974); 31 = Walraven et al. (1964); 32 = Lloyd Evans (1968); 33 = Feast (1967); $34=$ Stibbs (1955); $35=$ Jacobsen \& Wallerstein (1981); 36 = Taylor \& Booth (1998); 37 = Petterson et al. (2005); $38=$ Lloyd Evans (1980a); $39=$ Coulson \& Caldwell (1985); $40=$ Evans \& Lyons (1992); 41 = Abt \& Levy (1978); 42 = Sanford (1935); 43 = Evans et al. (1990); 44 = Szabados (1991); 45 = Szabados (1977).

data to see if there are clear outliers in the data or clear off-sets between datasets. Table 1 lists between parenthesis any off-set applied. Some datasets were in the end not considered at all because of very large error bars (see Table 2). The rms in the fit for each dataset separately was determined and this was used as typical error bar in a data point (unless the rms was consistent with the original error bars quoted in which case these were retained).

$K$-band data on the CIT/CTIO system was transformed to the SAAO system using the formula in Carter (1990) and Johnson IR-photometry was transformed according to Glass (1985).

A few stars in the sample are known or suspected binaries. Using the available $R V$ data, the orbits were redetermined for FF Aql, W Sgr and X Sgr, while for Y Oph we confirm Evans \& Lyons (1986) suggestion that there is little evidence for orbital motion. Details will be published elsewhere. The observed $R V$ were corrected for the orbital motion to leave the $R V$ variation due to the pulsation.

Regarding the interferometric data, limb darkened (LD) angular diameters for delta Cep were taken from Mérand et al. (2005), in preference over the less accurate data from Nordgren et al. (2002); for 1 Car from Kervella (2004c); for beta Dor, X Sgr, W Sgr from Kervella et al. (2004a); for zeta Gem, eta Aql from Kervella et al. (2004a) and Nordgren et al. (2002); for Y Oph from Kervella et al. (2004a) and Mérand et al. (2007). The Kervella et al. papers quote random and systematic error bars and they have been added quadratically.

The recent discovery of circumstellar emission (CSE) has raised the issue of whether the previously derived angular diameters are biased. For delta Cep, Mérand et al. (2006) conclude that the bias in angular diameter is less than $1 \%$ in the $K$-band, but find that a contribution of $1.5 \%$ in $K$-band flux due to an extended envelope improves the fit to the visibility curves. Similarly, for 1 Car, Kervella et al. (2006) conclude that the bias in angular diameter is of the order $0.6 \%$ in the $K$-band, but find that a contribution of about $4 \%$ in $K$-band flux due to an extended envelope improves the fit to the visibility curves.

For Y Oph the situation is more complicated. Mérand et al. (2007) find a contribution in the $K$-band of $5 \pm 2 \%$ in flux due to the CSE. Depending on this number and the value of the measured visibilities the uniform disk (UD) angular diameters have to be corrected. Their recommendation was followed to correct their CHARA/FLUOR measurements by $2 \%$ (a systematic error of $2 \%$ on the angular diameter was assumed due to the uncertainty in the level of circumstellar emission), and to correct the VINCI UD measurements of Kervella et al. (2004a) by $10 \%$.

For the other stars in our sample no information on the possible presence of CSE is available and no correction to the published LD diameters was made.

\section{The model}

The (corrected) $V$-, $K$ - and $R V$ data with error bars are fitted with a function of the form:

$F(t)=F_{0}+\sum_{i=1}^{i=N}\left(A_{i} \sin \left(2 \pi t \mathrm{e}^{i f}\right)+B_{i} \cos \left(2 \pi t \mathrm{e}^{i f}\right)\right)$

where $P=\mathrm{e}^{-f}$ is the period (in days). Typically, $P$ is determined from the fit to the available optical photometry as this dataset is usually most extensive. The period is then fixed when fitting Eq. (1) to the $K$-band and $R V$ data.

The determination of the parameters is done using the MRQMIN routine (using the Levenberg-Marquardt method) from Press et al. (1992) written in Fortran77, which minimises

$\chi^{2}=\sum_{i=1}^{i=n}\left(F_{i}-F\left(t_{i}\right)\right)^{2} /\left(\sigma_{F_{i}}\right)^{2}$,

with $F_{i}$ the measurement at time $t_{i}$ which has an error bar $\sigma_{F_{i}}$. Also the reduced $\chi^{2}$ is defined:

$\chi_{\mathrm{r}}^{2}=\frac{\chi^{2}}{(n-p)}$

and the quantity

$\mathrm{BIC}=\chi^{2}+(p+1) \ln (n)$, 
Table 2. Stars analysed to obtain the $p$-factor.

\begin{tabular}{ccccccc}
\hline \hline Name & $d$ & $A_{\mathrm{V}}$ & Data not used & Period $(\mathrm{d})$ & $p$ & $R\left(\mathrm{R}_{\odot}\right)$ \\
\hline delta Cep & $273 \pm 11$ & $0.23 \pm 0.11$ & $\mathrm{MJD}<43500, R V=3$ & 5.3662496 & $1.245 \pm 0.030 \pm 0.050$ & $42.52 \pm 0.038 \pm 1.72$ \\
1 Car & $498 \pm 50$ & $0.17 \pm 0.01$ & $\mathrm{MJD}<46500$ & 35.556274 & $1.193 \pm 0.058 \pm 0.120$ & $157.8 \pm 0.6 \pm 15.9$ \\
zeta Gem & $360 \pm 23$ & $0.06 \pm 0.03$ & $R V=3$ & 10.149888 & $1.320 \pm 0.104 \pm 0.084$ & $65.24 \pm 0.20 \pm 4.17$ \\
beta Dor & $318 \pm 16$ & $0.25 \pm 0.05$ & $R V=13$ & 9.8425589 & $1.254 \pm 0.430 \pm 0.063$ & $64.71 \pm 0.90 \pm 3.25$ \\
W Sgr & $439 \pm 38$ & $0.37 \pm 0.03$ & $R V=2,3$ & 7.5949292 & $1.660 \pm 0.862 \pm 0.140$ & $61.0 \pm 1.6 \pm 5.2$ \\
X Sgr & $333 \pm 20$ & $0.58 \pm 0.10$ & & 7.0127477 & $0.925 \pm 0.898 \pm 0.055$ & $52.68 \pm 0.85 \pm 3.17$ \\
& & & & & & \\
eta Aql & $274.1 \pm 2.1 \pm 12.9$ & $0.14 \pm 0.01$ & $R V=3$ & 7.1768136 & $1.640 \pm 0.231 \pm 0.078$ & $52.6 \pm 2.5$ \\
Y Oph & $691 \pm 5.2 \pm 24$ & $0.66 \pm 0.03$ & $\mathrm{MJD}<40000, R V=3$ & 17.1261444 & $1.706 \pm 0.328 \pm 0.081$ & $95.6 \pm 4.5$ \\
\hline
\end{tabular}

where $p=2 N+2$ is the number of free parameters $(p=2 N+1$ when fitting the $R V$ and $K$ light curve). As the number $N$ of harmonics to be fitted to the data is apriori not known, one could obtain ever better fits (lower $\chi^{2}$ ) by increasing $N$. The Bayesian information criterion (Schwarz 1978) is a formalism that penalises this, and $N$ (for the $V, K$ and $R V$ curve independently) is chosen such that BIC reaches a minimum.

Given the analytical form of Eq. (1), the radial velocity curve can be integrated exactly to obtain the variation in radius as a function of time (phase):

$\Delta R(t, \delta \theta)=-p \int_{t_{0}}^{t+P \delta \theta}\left(v_{\mathrm{R}}-\gamma\right) \mathrm{d} t$,

where $\gamma$ is the systemic velocity, $v_{\mathrm{R}}$ the radial velocity, $p$ the projection factor and $\delta \theta$ allows for a phase shift between the $R V$ curve and the angular diameter variations measured interferometrically or via the SB relation.

Then, the equation

$\theta(t)=9.3038 \operatorname{mas}\left(\frac{R_{0}+\Delta R(t, \delta \theta)}{d}\right)$

is fitted using the MRQMIN routine, with $\theta$ the angular diameter in mas, $R_{0}$ the stars radius in solar radii and $d$ the distance in pc. With interferometrically determined angular diameters and stars with known distance, the parameters $p, R_{0}$ and $\delta \theta$ are solved. When $R_{0}$ is known, one solves $p, d$ and $\delta \theta$.

In Sect. 6 the SB $(V-K)$ colour relation is discussed. An SB relation can be defined as follows (see van Belle 1999):

$\theta_{o}=\theta \times 10^{\left(m_{1} / 5\right)}$,

where $\theta$ is the LD angular diameter (in mas), and $m_{1}$ a dereddened magnitude (for example, $V$ ). The logarithm of this quantity (the zero magnitude angular diameter) is plotted against a de-reddened colour (for example, $\left.(V-K)_{0}\right)$,

$\log \theta_{0}=a \times\left(m_{2}-m_{3}\right)+b$.

The aim is to determine the coefficients $a$ and $b$.

\section{The period-radius relation}

Table 2 lists the results of the analysis. For the first five stars, Col. 2 gives the adopted distances with errors derived by Benedict et al. (2007). Column 4 lists which of the data in Table 1 were not used in the analysis. Column 5 lists the derived period, and Cols. 5 and 6 list the derived $p$-factor and mean radius. The first error bar is the error in the fit, while the second is the error due to the error in the distance.

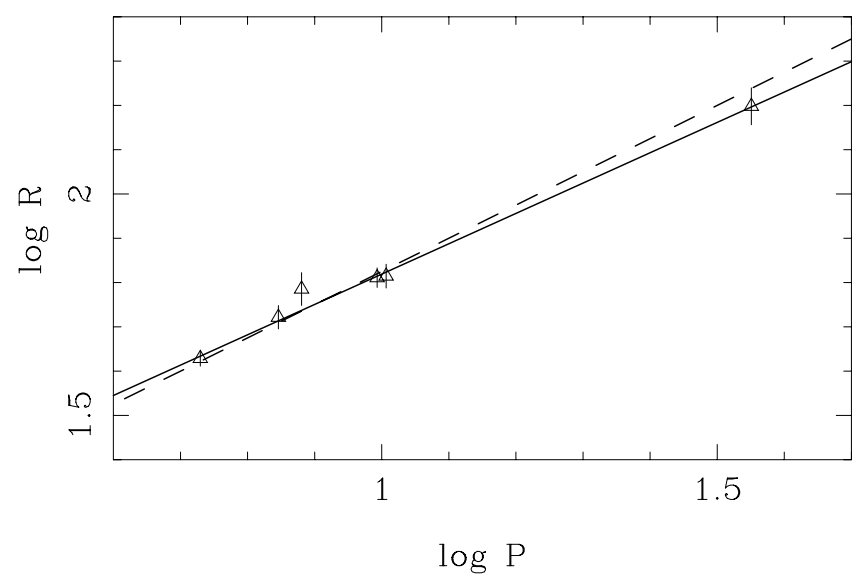

Fig. 1. PR relation derived in the present paper (the solid line which is the fit to the data points with error bars), compared to the PR relations of Gieren et al. (1998) and Laney \& Stobie (1995) represented by the dashed line.

The analysis of these five stars allows one to derive a PR relation and this is shown in Fig. 1. A linear weighted least-squares fit results in:

$\log R=(0.686 \pm 0.036) \log P+(1.134 \pm 0.034), \sigma=0.020$

where the two error bars for $R$ listed in Table 2 have been added quadratically.

This relation can be compared to recent reports in Gieren et al. (1998), $\log R=0.750 \log P+1.075(\sigma=0.036)$, which is almost identical to that in Laney \& Stobie (1995), $\log R=$ $0.751 \log P+1.070(\sigma=0.051)$, and that of Kervella et al. (2004c), $\log R=0.767 \log P+1.091$. These three relations have all been derived from a SB-type analysis and therefore had to assume a $p$-factor. Kervella et al. and Laney \& Stobie (1995) assumed a constant value of 1.36 , Gieren et al. use a value depending on period (see below). The shallower slope is likely related to the fact that there is no implicit assumption on the $p$-factor in deriving the PR relation. Interestingly, theory typically predicts slightly shallower slopes, e.g. $\log R=0.655 \log P+1.188$ (Bono et al. 1998) which is within the error bar derived here.

Using the distances in van Leeuwen et al. (2007), those based on averaging the data in Benedict et al. and the revised Hipparcos parallaxes, results in the PR relation: $\log R=(0.696 \pm$ $0.036) \log P+(1.114 \pm 0.034), \sigma=0.024$, which is statistically indistinguishable from Eq. (8).

\section{The projection factor}

Using the PR relation of Eq. (8), the radius of the last two stars in Table 2 were estimated with their error bar (last column), and the 


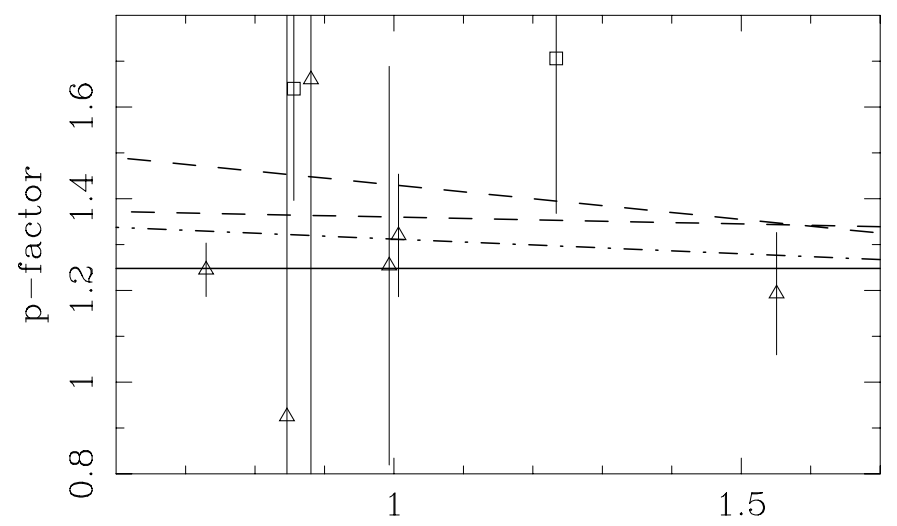

$\log \mathrm{P}$

Fig. 2. The $p$ factor plotted versus log period and the best fitting constant value of $p=1.27$. The open squares indicate the two stars for which the radius was fixed from the PR relation. The dashed lines represent the $p$-factor dependence proposed by Gieren et al. (1993, 2005), andc the dot-dashed line that by Nardetto et al. (2007), see text.

distance and $p$-factor determined with their error bars (internal, and due to the uncertainty in $R$ ).

Figure 2 plots the $p$-factor against $\log P$ for the seven stars analysed. For delta Cep the value derived here, $1.245 \pm 0.030 \pm$ 0.050 , is in excellent agreement with the $1.273 \pm 0.021 \pm 0.050$ in Mérand et al. (2005). Both papers use the same interferometric dataset and essentially the same distance (273 versus $274 \mathrm{pc}$ ) and differ only in the used RV dataset and the fitting of the $R V$ curve (Fourier series versus periodic cubic spline).

The quoted error bars on $p$ were added quadratically, and a weighted least-squares fit is made to find that there is no evidence of a dependence on period: $p=(1.28 \pm 0.15)-(0.01 \pm$ $0.16) \log P$. The best fitting constant value is, $p=1.27 \pm 0.05$. The two stars were $R$ was fixed rather than $d$ both have $p$-factors above this mean value. Excluding these from the solution makes little difference. The fit including period becomes $p=(1.29 \pm$ $0.07)-(0.04 \pm 0.08) \log P$, the constant becomes $p=1.25 \pm 0.05$.

Had I adopted the PR relation from Gieren et al. (1998), the radii would have been $100 \pm 4.7$ and $52.1 \pm 2.5 \mathrm{R}_{\odot}$ for $\mathrm{Y}$ Oph and eta Aql, respectively, and the $p$-factor $1.78 \pm 0.34 \pm 0.09$ and $1.62 \pm 0.23 \pm 0.08$ respectively. The choice of the PR relation has therefore no impact on the result of the best fit value of $p$.

Using the distances in van Leeuwen et al. (2007), those based on averaging the data in Benedict et al. and the revised Hipparcos parallaxes, gives much the same result with a non-significant slope of $(-0.02 \pm 0.12)$ and a best-fitting constant of $1.25 \pm 0.04$.

The low value of $p$ is in contrast with values commonly adopted and suggested, e.g. the $p=1.39-0.03 \log P$ used in the Gieren et al. papers (1993, 1997, 1998; Storm et al. 2004; Barnes et al. 2003), based on the work of Hindsley \& Bell (1986). Gieren et al. (2005) proposed an even stronger dependence on period, $p=1.58-0.15 \log P$, in order that the distance to the LMC derived from SB distances does not depend on period, and SB distances to Galactic cluster Cepheids are in agreement with ZAMS-fitting distances. The slope they find in this way hinges on a number of short period Cepheids in the LMC cluster NGC 1866 (Storm et al. 2005) for which however they adopt the historical reddening quoted in the literature which is different from that suggested recently by Groenewegen \& Salaris (2003).
The most recent theoretical models, specifically computed for delta Cep (Nardetto et al. 2004), suggest $p=1.27 \pm 0.01$ which is in very good agreement with the value derived here and by Mérand et al. (2005).

In a paper that was published during the submission stage of the present paper, Nardetto et al. (2007) present a careful theoretical analysis of the different ingredients involved in the $p$-factor, and favour the relation $p=1.376-0.064 \log P$ to be used in the SB-technique.

Their theoretical investigations suggest that there is a difference between the $p$-factor to be used with wide-band interferometry (like in the present study) and with $R V$ data (when applying the SB-technique). For delta Cep this difference is of the order of 0.06 (Nardetto et al. 2004, 2007). If such an off-set is applied then, statistically, the relations $p=1.33-0.03 \log P$ and $p=1.32-0.064 \log P$ are consistent with the data in Fig. 2, while a relation $p=1.52-0.15 \log P$ can be excluded at the $90 \%$ level.

\section{The surface-brightness relation}

Independently of the derivation of the PR relation or the $p$-factor the available interferometric, optical and infrared data can be used to calibrate the SB relation for Cepheids, very much in line with Kervella et al. (2004b). The influence of the presence of dust is taken into account, see later.

Based on the Fourier fits to the light curves, the $V$ and $K$-magnitude at the time of the interferometric measurements are determined. An error bar is assigned equal to the average of the error bars in the individual measurements, typically $0.015 \mathrm{mag}$.

The optical and infrared magnitudes have to be de-reddened. For the five stars with trigonometric parallaxes, the $A_{\mathrm{V}} \mathrm{s}$ and errors listed in Benedict et al. (2007) were adopted which are based on the photometry of reference stars in their astrometric field of view. For eta Aql and Y Oph the average $E(B-V)$ values listed in Laney \& Stobie (2007) were adopted.

Selective reddenings of $A_{\mathrm{V}}=3.3 E(B-V)$ and $A_{\mathrm{K}} / A_{\mathrm{V}}=$ 0.0909 were adopted. The discovery of CSE around Cepheids has an impact on their colours. For 1 Car (Kervella et al. 2006), delta Cep (Mérand et al. 2006) and Y Oph (Mérand et al. 2007) the fraction of CSE to the stellar flux has been estimated to be $4.2 \%, 1.5 \%, 5 \%$, respectively. For such a fraction $f$, the $K$ magnitudes have been corrected by $c_{\mathrm{K}}=+2.5 \log (1+f / 100)$. However, not only is $K$ affected by emission but $V$ as well because of the extinction due to the presence of dust. In a first order attempt to estimate this effect, a 1D dust radiative transfer program was used (Groenewegen 1993, also see Groenewegen 1998) with silicate grains condensing at $1800 \mathrm{~K}$ around an F6 star to estimate that for emission levels of a few percent in $K$, the $V$ magnitude should be corrected by $c_{\mathrm{V}}=-c_{\mathrm{K}} / 6$.

The dust model presented is clearly an oversimplification as it predicts the grains to condense at about 15 stellar radii, while the interferometric observations show that the infrared excess is present much closer to the star at 2-3 stellar radii, probably indicating that the mass-loss is non-uniform and/or non spherically symmetric.

The computations were done using the MRQMIN routine to fit the angular diameters directly instead of Eq. (8). This was done to allow fitting of the $E(B-V)$ per star as well. Figure 3 shows the result when keeping the extinction values fixed.

Numerically, the fitting relation is: $\log \theta_{0}=(0.2762 \pm$ $0.0011)(V-K)_{0}+(0.5223 \pm 0.0016)$ for a reduced chi-square $\chi_{\mathrm{r}}^{2}=3.48$. 


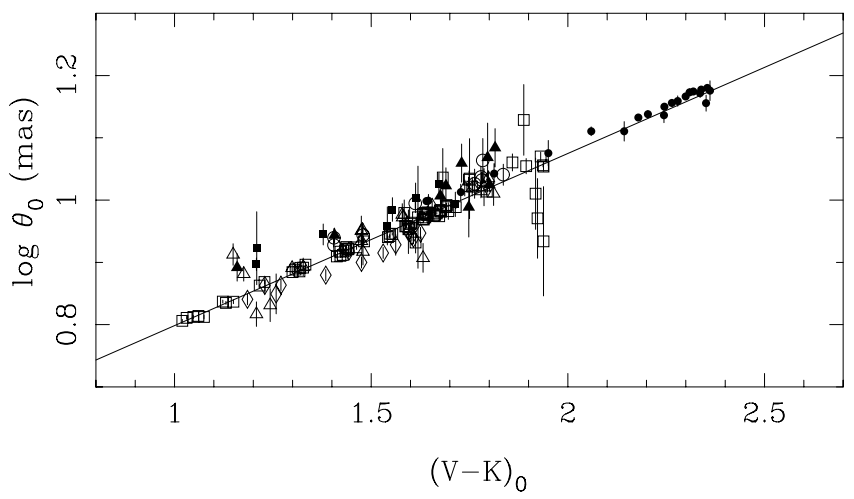

Fig. 3. Log (zero-magnitude angular diameter) versus de-reddened ( $V-$ $K)$ colour for Cepheids. Different symbols indicate the different stars.

A Monte-Carlo simulation was run to investigate the influence of the errors in the data, $E(B-V)$ and the influence of the CSE. Random numbers from a Gaussian distribution were drawn to generate new sets of $\theta, V$ and $K$, and $E(B-V)$. For $f$ a Poisson distribution was used, where the stars for which no information is available a value $f=0.5 \%$ was used. The fit becomes: $\log \theta_{0}=(0.2755 \pm 0.0045)(V-K)_{0}+(0.5234 \pm 0.0080)$, where the errors now come from the dispersion in the coefficients and are not the fit errors.

The solutions with the lowest $\chi_{\mathrm{r}}^{2}$ were inspected to see if these had extinction values systematically different from the adopted ones. This was only the case for Y Oph.

Solving for the 2 coefficients and $E(B-V)$ for Y Oph results in: $\log \theta_{0}=(0.2761 \pm 0.0011)(V-K)_{0}+(0.5228 \pm 0.0016)$, $E(B-V)=0.778 \pm 0.018$ with $\chi_{\mathrm{r}}^{2}=3.18$.

The Monte-Carlo simulation results in:

$\log \theta_{0}=(0.2752 \pm 0.0045)(V-K)_{0}+(0.5235 \pm 0.0092)$,

with $E(B-V)=0.78 \pm 0.05$ for Y Oph, which is the finally adopted relation in the present paper.

Changing the selective reddening coefficients to $A_{\mathrm{V}}=$ $3.1 E(B-V)$ and $A_{\mathrm{K}} / A_{\mathrm{V}}=0.12$ has little effect; the coefficients in Eq. (9) become $(0.2745 \pm 0.0045),(0.5222 \pm 0.0089)$. (1997):

This relation can be compared to those by Fouqué \& Gieren

$\log \theta_{0}=(0.262 \pm 0.004)(V-K)_{0}+(0.547 \pm 0.006)$,

and recently derived by Kervella et al. (2004b):

$\log \theta_{0}=(0.2672 \pm 0.0016)(V-K)_{0}+(0.5354 \pm 0.0012)$.

The agreement is very good, especially with Kervella et al.

The above SB relation with the constant $p$-factor can now be applied in a BW analysis to the stars FF Aql, RT Aur, Y Sgr and $\mathrm{T}$ Vul for which Benedict et al. list the trigonometric parallax but for which there are no interferometric observations which can be used in the calibration.

For these stars, the data is listed in Table 1 and the results in Table 3. Column 3 lists the derived period, and Cols. 4 and 5 the distance and $A_{\mathrm{V}}$ s from Benedict et al., which are adopted here. The last columns list the distance and radius derived from the non-linear weighted minimisation routine (LSF) and the linear bi-sector fit (BS), as used by Storm et al. (2004) and Gieren et al. (2005) for example. The error listed is the fit error to which a $4 \%$ error could be added due to the uncertainty in the adopted $p$. The fraction of CSE is set to $0 \%$ in all cases.
For every star there is second line which is the result of a Monte-Carlo simulation where for each simulation a new dataset is generated based on Gaussian errors on the individual $V, K, R V$ measurements, and where in the BW-analysis Gaussian errors on $A_{\mathrm{V}}$ and $p$ are considered.

Figure 4 shows in the top panel the relation between the angular diameter predicted by the SB-relation versus radial variation, and in the bottom panel the angular diameter as a function of phase. The lines are the best fit based on the LSF for $d$ and $R$.

Three of the four stars show a large scatter in the predicted angular diameters and this is due to the very poor infrared data available in the literature. In these cases the distance and radius from the bi-sector fit and the least-squares fit differ significantly. In this light it is difficult to interpret the agreement in the case of FF Aql, and the disagreement in the case of Y Sgr and RT Aur, between the trigonometric distance and that from the leastsquares fit using the SB technique. Interestingly, van Leeuwen et al. (2007) quotes a revised Hipparcos parallax for Y Sgr that will place it at $268 \pm 21 \mathrm{pc}$, i.e. considerably shorter than the distance in Benedict et al.

The best quality data amongst the four stars is available for $\mathrm{T}$ Vul. Least-squares and bi-sector fit agree, and agree within 1sigma of the Benedict et al. distance. If a larger $p$-factor of 1.33 had been adopted (following the discussion in Nardetto et al. 2004 and at the end of Sect. 5) the agreement would even be better.

\section{Summary and discussion}

The last few years have seen developments relevant for a better understanding of, and more accurate application of, the BaadeWesselink method using SB relations. These are the availability of accurate trigonometric parallaxes, and the progress in interferometry which allows for accurate determination of angular diameters, and which also led to a better understanding of the circumstellar emission around Cepheids.

In the present paper, I use the currently available data to determine the SB $(V-K)$ colour relation taking into account in a simple way the effect of the circumstellar emission. The best fitting relation is very close to that found by Kervella et al. (2004b) even though additional interferometric measurements and the effect of CSE have been taken into account. The Monte-Carlo simulation which allows for the errors in the interferometric, $V$ and $K$ data, the extinction, and the level of CSE shows that the error bars are slightly larger than one is led to believe from the fit alone.

The most important conclusion from this work is that the currently available data suggests that the $p$-factor may not be a function of period, and may be assumed to be constant, $p=$ $1.27 \pm 0.05$.

This value is smaller than that often adopted in the literature, but this could be related to a difference in $p$-factor applicable to interferometry and RV data (see Nardetto et al. 2004), which is of the order of 0.06. Allowing for this offset, relations in the literature with a shallow dependence of the $p$-factor on slope are consistent with the data.

A smaller $p$ factor implies smaller distances in connection with the SB-technique and this has implications for the distance to the LMC. Gieren et al. (2005) using $p=1.58-0.15 \log P$ find a distance modulus to the LMC of $18.56 \pm 0.04$. Although a full analysis is beyond the scope of this paper, the mean period of the LMC cepheids in their analysis has $\log P$ about 1 , so with a constant $p$-factor, as advocated here, one may expect an LMC distances shorter by about $0.12 \mathrm{mag}$. 
Table 3. Stars analysed to obtain the distance.

\begin{tabular}{ccccccccc}
\hline \hline Name & Data not used & Period $(\mathrm{d})$ & $d(\mathrm{pc})$ & $A_{\mathrm{V}}$ & $d(\mathrm{LSF})$ & $d(\mathrm{BS})$ & $R(\mathrm{LSF})$ & $R(\mathrm{BS})$ \\
\hline Y Sgr & $R V=3$ & 5.7650394 & $469 \pm 63$ & $0.67 \pm 0.04$ & $353.1 \pm 35.4$ & $291.5 \pm 25.2$ & $31.5 \pm 3.2$ & $25.9 \pm 2.2$ \\
& & & & & $356.8 \pm 43.3$ & $255.0 \pm 22.6$ & $31.8 \pm 3.9$ & $22.6 \pm 2.1$ \\
FF Aql & $R V=3, V=7$ & 4.4708958 & \multirow{3}{*}{$356 \pm 23$} & $0.64 \pm 0.06$ & $366.7 \pm 71.8$ & $183.6 \pm 25.6$ & $34.0 \pm 6.7$ & $17.1 \pm 2.4$ \\
& & & & & $366.9 \pm 91.4$ & $134.2 \pm 23.7$ & $34.1 \pm 8.6$ & $12.5 \pm 2.2$ \\
RT Aur & & 3.7283149 & \multirow{2}{*}{$417 \pm 33$} & $0.20 \pm 0.08$ & $338.1 \pm 34.4$ & $257.4 \pm 25.6$ & $24.8 \pm 2.5$ & $18.9 \pm 1.9$ \\
& & & & $332.7 \pm 37.0$ & $213.7 \pm 19.5$ & $24.3 \pm 2.8$ & $15.6 \pm 1.4$ \\
T Vul & $R V=2, K=21$ & 4.4354167 & \multirow{2}{*}{$526 \pm 64$} & $0.34 \pm 0.06$ & $476.8 \pm 13.1$ & $471.0 \pm 09.2$ & $30.9 \pm 0.9$ & $30.6 \pm 0.6$ \\
& & & & & $475.6 \pm 22.0$ & $463.4 \pm 20.8$ & $30.8 \pm 1.4$ & $30.0 \pm 1.3$ \\
\hline
\end{tabular}

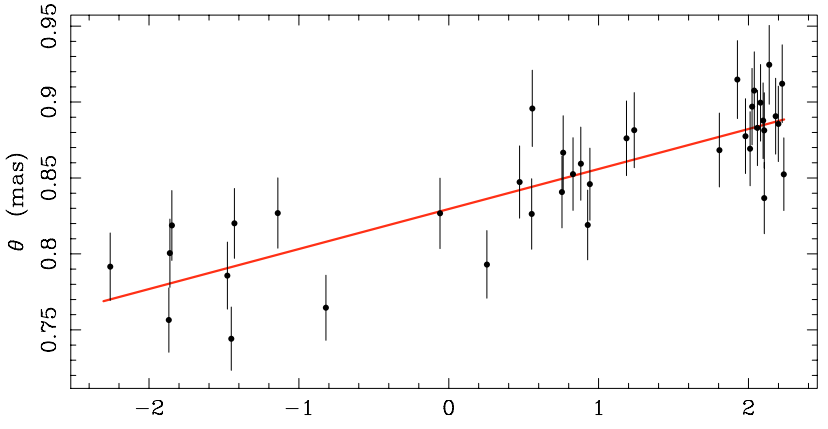

$\Delta \mathrm{R}$ (solar radii)
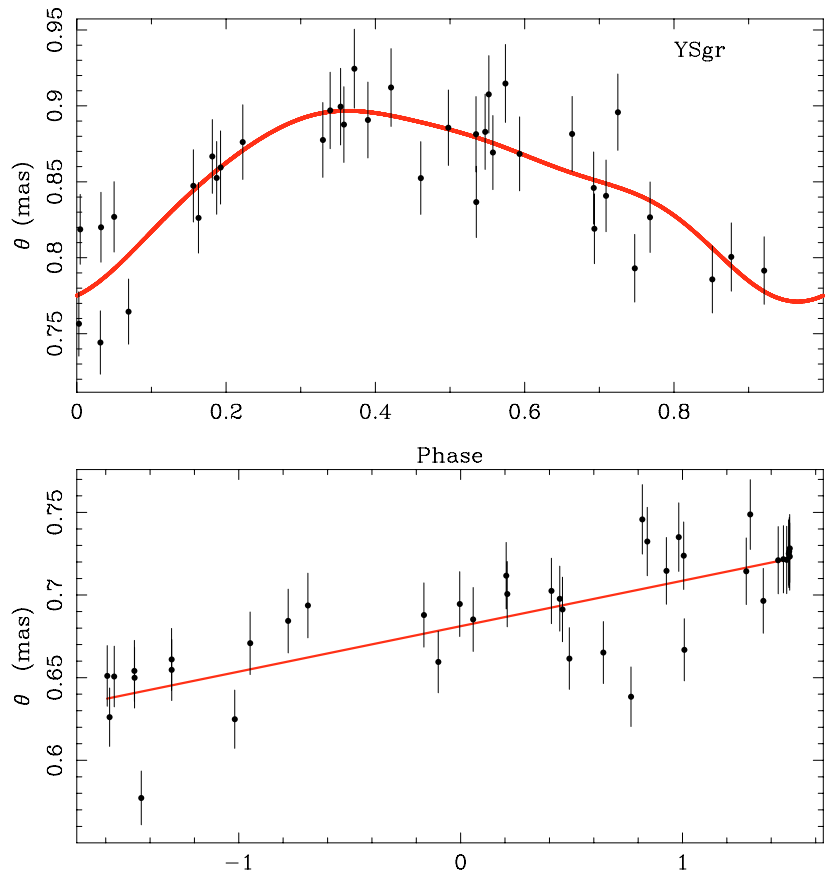

$\Delta \mathrm{R}$ (solar radii)

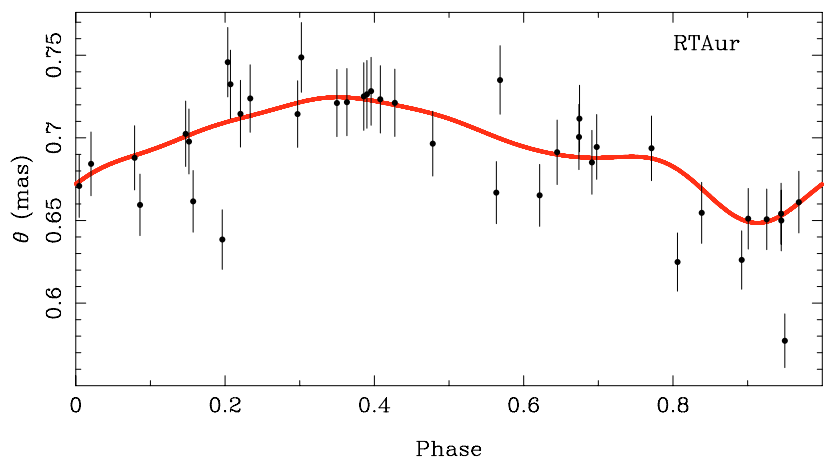

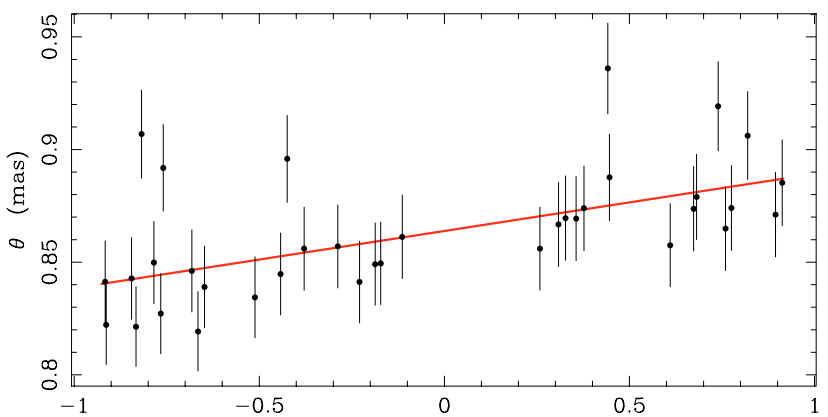

$\Delta \mathrm{R}$ (solar radii)
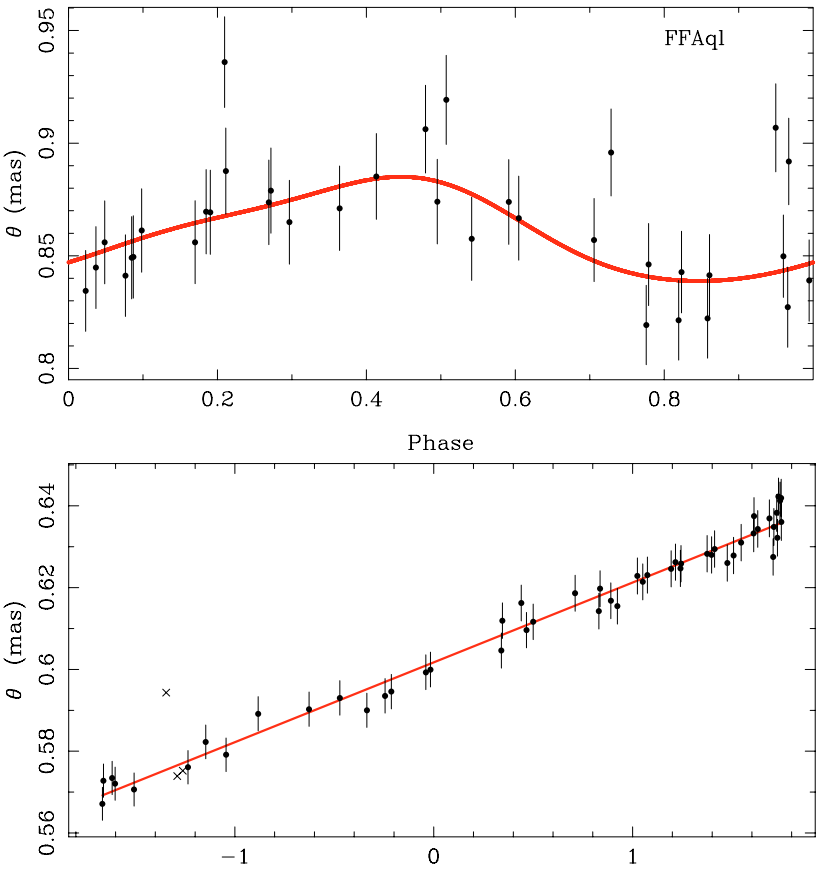

$\Delta \mathrm{R}$ (solar radii)

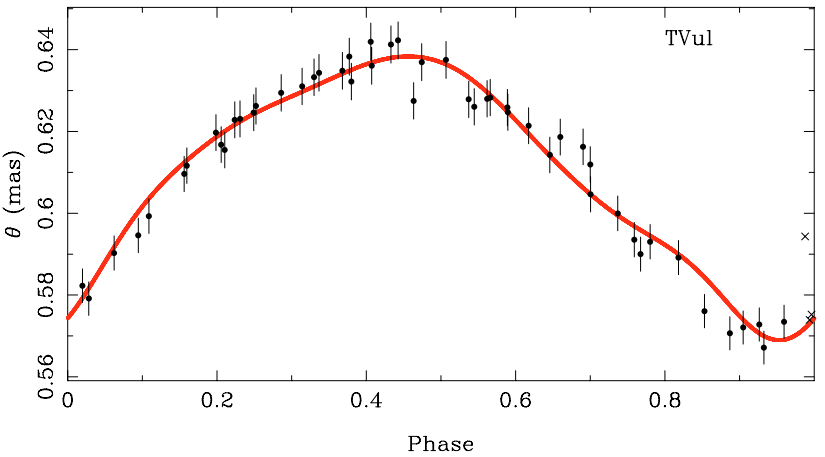

Fig. 4. Data points show the photometrically determined angular diameters and the best fit to these points. Crosses indicate datapoints not taken into account in the fit. 
Acknowledgements. This research has made use of the SIMBAD database, operated at CDS, Strasbourg, France.

\section{References}

Abt, H. A., \& Levy S. G., 1978, PASP, 90, 188

Albrow, M. D., \& Cottrell, P. L. 1996, MNRAS, 280, 917

Babel, J., Burki, G., Mayor, M., Chmielewski, Y., \& Waelkens, C. 1989, A\&A, 216,125

Barnes, T. G., Moffett, T. J., \& Slovak, M. H. 1987, ApJS, 65, 307

Barnes, T. G., Fernley, J. A., Frueh, M. L., et al. 1997, PASP, 109, 645

Barnes, T. G., Jeffery, E. J., Berger, J. O., et al. 2003, ApJ, 592, 539

Barnes, T. G., Jeffery, E. J., Montemayor, T. J., \& Skillen, I. 2005, ApJS, 156, 227

Benedict, G. F., McArthur, B. E., Fredrick, L. W. et al. 2002, AJ, 124, 1695

Benedict, G. F., McArthur, B. E., Feast, M. W. et al. 2007, AJ, 133, 1810

Berdnikov, L. N., Dambis, A. K., \& Vozyakovs, O. V. 2000, A\&AS, 143, 211

Bersier, D. 2002, ApJS, 140, 465

Bersier, D., Burki, G., Mayor, M., \& Duquennoy, A. 1994, A\&AS, 108, 25

Bono, G., Caputo, F., \& Marconi, M. 1998, ApJ, 497, L43

Caldwell, J. A. R., Coulson, I. M., Dean, J. F., \& Berdnikov, L. N. 2001, JAR, 7, 4

Carter, B. S. 1990, MNRAS, 242, 1

Coulson, I. M., \& Caldwell, J. A. R. 1985, SAAO Circ., 9, 5

Evans, N. R., \& Lyons, R. 1986, AJ, 92, 436

Evans, N. R., Welch, D. L., Scarfe, C. D., \& Teays, T. J. 1990, AJ, 99, 1598

Feast, M. W., 1967, MNRAS, 136, 141

Fouqué, P., \& Gieren, W. P. 1997, A\&A, 320, 799

Gieren, W. P., Barnes T. G., \& Moffett, T. J. 1993, ApJ, 418, 135

Gieren, W. P., Fouqué, P., \& Gómez, M. 1997, ApJ, 488, 74

Gieren, W. P., Fouqué, P., \& Gómez, M. 1998, ApJ, 496, 17

Gieren, W. P., Storm, J., Barnes, T. G., et al. 2005, ApJ, 627, 224

Glass, I. S. 1985, IrAJ, 17, 1

Gorynya, N. A., Samus', N. N., Sachkov, M. E., et al. 1998, PAZh, 24, 939 (VizieR On-line Data Catalog: III/229)

Groenewegen, M. A. T. 1993, Ph.D. Thesis, Chapter 5, University of Amsterdam Groenewegen, M. A. T. 1995, A\&A, 293, 463

Groenewegen, M. A. T., \& Salaris, M. 2003, A\&A, 410, 887

Hindsley, R. B., \& Bell, R. A. 1986, PASP, 98, 881

Jacobsen, T. S. 1974, ApJ, 191, 691

Jacobsen, T. S., \& Wallerstein, G. 1981, PASP, 93, 481

Jacobsen, T. S., Wallerstein, G., \& Abt, H. A. 1984, PASP, 96, 630

Kervella, P., Nardetto, N., Bersier, D., Mourard D., et al. 2004a, A\&A, 416, 941

Kervella, P., Bersier, D., Mourard D., Nardetto, N., Fouqué, P., \& Coudé du Foresto, V. 2004b, A\&A, 428, 587

Kervella, P., Bersier, D., Mourard D., Nardetto., N., \& Coudé du Foresto, V. 2004c, A\&A, 423, 327

Kervella, P., Fouqué, P., Storm, J. et al. 2004d, ApJ, 604, L113
Kervella, P., Mérand, A., Perrin, G., \& Coudé du Foresto, V. 2006, A\&A, 448, 623

Kimeswenger, S., Lederle, C., Richichi, A. et al. 2004, A\&A, 413, 1037

Kiss, L. L. 1998, MNRAS, 297, 825

Kiss, L. L. 2000, MNRAS, 314, 420

Laney, C. D., \& Stobie, R. S. 1992, A\&AS, 93, 93

Laney, C. D., \& Stobie, R. S. 1995, MNRAS, 274, 337

Laney, C. D., \& Stobie, R. S. 2007, MNRAS, 377, 147

Lenz, P., \& Breger, M. 2005, CoAst, 146, 53

Lloyd Evans, T. 1968, MNRAS, 141, 109

Lloyd Evans, T. 1980a, SAAO Circ., 1, 163

Lloyd Evans, T. 1980b, SAAO Circ., 1, 257

Madore, B. F. 1975, ApJS, 29, 219

Mérand, A., Kervella, P., Coudé du Foresto, V., et al. 2005, A\&A, 438, L9

Mérand, A., Kervella, P., Coudé du Foresto, V., et al. 2006, A\&A, 453, 155

Mérand, A., Aufdenberg, J., Kervella, P., et al. 2007, ApJ, 664, 1093

Moffett, T. J., \& Barnes, T. G. 1984, ApJS, 55, 389

Nardetto, N., Fokin, A., Mourard, D., et al. 2004, A\&A, 428, 131

Nardetto, N., Mourard, D., Kervella, P., et al. 2006, A\&A, 453, 309

Nardetto, N., Mourard, D., Mathias, Ph., Fokin, A., \& Gillet, D. 2007, A\&A, 471,661

Nordgren, T. E., Lane, B. F., Hindsley, R. B., \& Kervella, P. 2002, AJ 123, 3380

Pel, J. W. 1976, A\&AS, 24, 413

Petterson, O. K. L., Cottrell, P. L., \& Albrow, M. D. 2004, MNRAS, 350, 95

Petterson, O. K. L., Cottrell, P. L., Albrow, M. D., \& Fokin, A. 2005, MNRAS, 362,1167

Sanford, R. F. 1935, ApJ, 81, 140

Schwarz, G. 1978, Ann. Stat., 6, 461

Shobbrook, R. R. 1992, MNRAS, 255, 486

Stibbs, D. W. N. 1955, MNRAS, 115, 363

Storm, J., Carney, B. W., Gieren, W. P., et al. 2004, A\&A, 415, 531

Storm, J., Gieren, W. P., Fouqué, P., Barnes, T. G., \& Gómez, M. 2005, A\&A, 440,487

Szabados, L. 1977, Mitt. Sternw. ung. Akad. Wiss., Budapest, 70

Szabados, L. 1980, Commun. Konkoly Obs. Hung. Acad. Sci., Budapest, 76

Szabados, L. 1981, Commun. Konkoly Obs. Hung. Acad. Sci., Budapest, 77

Szabados, L. 1991, Commun. Konkoly Obs. Hung. Acad. Sci., Budapest, 96

Szabados, L. 2003, Inf. Bull. Var. Stars, 5394

Taylor, M. M., \& Booth, A. J. 1998, MNRAS 298, 594

Taylor, M. M., Albrow, M. D., Booth, A. J., \& Cottrell, P. L. 1997, MNRAS, 292, 662

van Belle, G. T., Lane, B. F., \& Thompson, R. R. 1999, AJ, 117, 521

van Leeuwen, F., Feast, M. W., Whitelock, P. A., \& Laney, C. D. 2007, MNRAS, 379,723

Walraven, J. H., Tinbergen, J., \& Walraven, T. 1964, BAN, 17(7), 520

Welch, D. L., Wieland, F., McAlary, C. W., et al. 1984, ApJS, 54, 547

Wilson, T. D., Carter, M. W., Barnes, T. G., Van Citters, G. W., \& Moffett, T. J. 1989, ApJS, 69, 951

Wisniewski, W. Z., \& Johnson, H. R. 1968, CoLPL 7, 57 